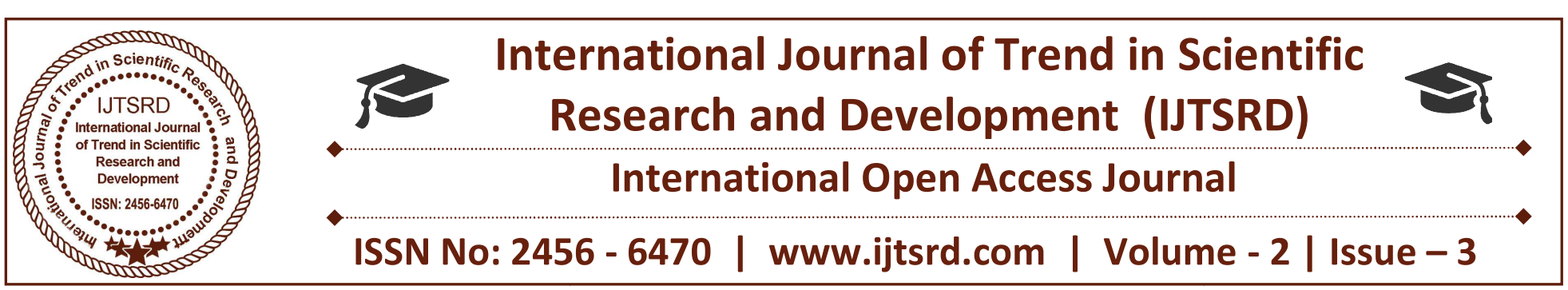

\title{
Hot Spot Temperature Analysis of Transformer using FEM on COMSOL
}

\author{
Swapnil Solanki $^{1}$, Rohit Jangid ${ }^{2}$, Gaurav Srivastava ${ }^{3}$, Prateek Sharma ${ }^{4}$, Ritvik Chaturvedi ${ }^{5}$ \\ ${ }^{3}$ Assistant Professor \\ Department of Electrical Engineering, Poornima College \\ of Engineering, Sitapura, Jaipur, Rajasthan, India
}

\section{ABSTRACT}

The transformers are static bits of mechanical assembly with at least two windings, which by electromagnetic enlistment; change an arrangement of substituting voltage and current into another arrangement of voltage and current for the most part of various esteems and at same recurrence for transmitting electrical power. In a transformer task, a piece of the electrical vitality is changed over into the warmth. That is the reason the exact estimation of temperatures in basic focuses is of down to earth intrigue. Problem area temperature (HST) esteem is a noteworthy factor that straightforwardly influences the task of transformers [1].

Keywords: Finite Element Method (FEM), Magnetic Flux, HST, Transformer, COMSOL

\section{INTRODUCTION}

Transformers speak to the biggest segment of capital interest in transmission and dissemination substations. In addition, transformer blackouts have a significant monetary effect on the activity of an electrical system. A problem area temperature calculation for computing the problem area temperature of a specifically stacked transformer, utilizing information acquired in a short out warming test, is given. These papers propose changes in the displaying of warm procedures inside the transformer tank. Estimation strategies must be founded on a vitality adjust condition. A few endeavors of warmth exchange hypothesis comes about application to the warmth exchange from twisting to oil. The utilization of normal warmth exchange coefficient is run of the mill in a transformer planning procedure to compute required number (region) of cooling surfaces. In this paper, a method for acquiring the temperature conveyance in the transformer is proposed [2]. For numerical reproduction of specified condition, it has been utilized limited component strategy. That is the reason the exact estimation of temperatures in basic focuses is of commonsense intrigue. Warm effect leads not exclusively to long haul oil/paper protection debasement; it is likewise a constraining variable for the transformer task. In this way, the learning of the temperature, particularly the problem area temperature, is of high premium.

\section{Hot Spot Temperature}

Stacking capacity of energy transformers is restricted for the most part by winding temperature. As a major aspect of acknowledgment tests on new units, the temperature rise test is planned to exhibit that, at full load and appraised surrounding temperature, the normal winding temperature will not surpass the points of confinement set by industry benchmarks. In any case, the temperature of the winding is not uniform and the genuine constraining component is really the most blazing area of the twisting regularly called winding problem area. This problem area region is found some place toward the highest point of the transformer, and not open for coordinate estimation with traditional techniques. The temperature of strong protection is the principle factor of transformer maturing. With temperature and time, the cellulose protection experiences a DE polymerization process. As the cellulose chain gets shorter, the mechanical properties of paper, for 
example, rigidity and flexibility debase [3]. In the long run the paper winds up weak and isn't fit for withstanding short out powers and even ordinary vibrations that are a piece of transformer life. This circumstance describes the finish of life of the strong protection. Since it is not reversible, it likewise characterizes the transformer end of life.

This procedure is outstanding to transformer proprietors and supported endeavors have been made to screen the problem area temperature to exploit cool encompassing temperature, broaden the transformer life while giving crisis over-burdening abilities and exploiting market openings. The appraised problem area temperature is $110^{\circ} \mathrm{C}$.

It has seen that an expansion of $7^{\circ} \mathrm{C}$ will twofold the maturing speeding up factor. For more seasoned transformer, the evaluated problem area temperature is either $95^{\circ} \mathrm{C}$ or $97^{\circ} \mathrm{C}$. It is additionally extremely touchy to temperature and if there should arise an occurrence of crisis (accepting a problem area temperature of $140^{\circ} \mathrm{C}$ ); the maturing speeding up factor is around 100, which implies one hour in this condition is identical to 100 hours at the appraised temperature.

It is noticed that the customary problem area temperature computation technique utilizes various suppositions that are not right:

$>$ Oil temperature in the cooling pipe is expected the same as the best oil temperature.

$>$ The adjustment in twisting protection with temperature is dismissed.

$>$ The adjustment in oil consistency with temperature is disregarded.

$>$ The impact of tap position is dismissed.

$>$ The variety of encompassing temperature is accepted to immediaty affect oil temperature [4].

\section{Finite Element Method}

This problem area territory is found some place toward the highest point of the transformer, and not available for coordinate estimation with traditional strategies considering the impact of relocation current $\mathrm{T}-\Omega$ technique is received to computing the stray misfortunes in metal auxiliary parts of influence transformer.

$$
\mathrm{E}=\sigma+\varepsilon(\partial-1) / \partial t(\nabla . T)
$$

The above equation is in eddy domains

\section{FEM FORMULATION EQUATIONS}

Coupled field limited component strategy is a numerical methodology for getting limit estimations of scientific model for our situation that would be a transformer show. The standard of the technique is to supplant a constant area by number of sub areas, which are normally alluded to as components whose conduct is displayed by addition work containing a couple of obscure esteems at the hubs of the component. The variable esteems and states of transformers have taken as the spaces of the model. In assessing numerical model, these following advances are critical.

\section{1) Discretization of the space}

2) Induction of the component conditions

3) Gathering of the components

4) Arrangement of the arrangement of conditions

The way in which the area is discretized will influence the PC stockpiling necessity, the preparing time, and additionally exactness of the numerical outcomes. High request capacities, albeit more exact, yet in addition more entangled plan. Thus, a straightforward and fundamental direct insertion is still generally utilized.

The articulation for the obscure arrangement in a component, say component $\mathrm{e}$, in the accompanying structure

$$
\emptyset^{\mathrm{le}}=\sum(\mathrm{j}=1, \mathrm{n}) N_{\mathrm{j}}^{\mathrm{e}} \emptyset_{\mathrm{j}}^{\mathrm{e}}
$$

Where $\mathrm{n}$ is number of hubs in the component, $\varnothing$ ?e the field an incentive at hub $\mathrm{j}$ and the extension work which is otherwise called fundamental capacity or shape work. A first acknowledgment of a transient 3D FE transformer demonstrate utilizing COMSOL Multiphysics 5.3a, generous model advances, most eminently in the usage of non-straight attractive materials, the thought of whirlpool streams and the fuse of a warm model. The electromagnetic FE demonstrate registers the associations between the electrical subsystem on the essential and auxiliary sides and the attractive subsystem. Ampère's circuital law and Faraday's law of enlistment depict them.

The summed up arrangement of non-homogeneous warmth conduction condition with non-homogeneous limit condition, in Cartesian facilitate framework is composed in this manner: 


$$
k \cdot\left(\frac{\partial^{2} T}{\partial x^{2}}+\frac{\partial^{2} T}{\partial y^{2}}\right)+Q=0
$$

In the district $\mathrm{a}<\mathrm{x}<\mathrm{b}$ and $\mathrm{zero}<\mathrm{y}<1$. At the internal tube shaped surface:

$-k_{1} \frac{\partial T}{\partial x}+h_{1} T=f_{1}(y)$

At the external tube shaped surface:

$$
k_{2} \frac{\partial T}{\partial x}+h_{2} T=f_{2}(y)
$$

At the base level surface:

$$
-k_{3} \frac{\partial T}{\partial y}+h_{3} T=f_{3}(x)
$$

$$
f_{4}(x)=h_{4} T_{\text {top }}
$$

Largely, warm conductivities are demonstrated extraordinary, and are shown in like manner. Nevertheless, in a genuine case, the warm conductivities in outspread ways ( $\mathrm{k} 1$ and $\mathrm{k} 2$ ) are equivalent and conductivities pivotal way will likewise be the same (k3 andk4). Warm conductivity has been dealt with as a vector amount, having segments in both outspread and pivotal course.

Resultant warm conductivity of the framework can be evaluated as:

$$
K=\sqrt{k_{r}^{2}+k_{z}^{2}}
$$

At the best level surface:

$$
k_{4} \frac{\partial T}{\partial y}+h_{4} T=f_{4}(x)
$$

Conditions (1) through (5) speak to the general warmth conduction condition with convection at all four limit surfaces. In the above conditions, temperature $\mathrm{T}$ is a component of room factors $\mathrm{x}$ and $\mathrm{y}$. The term $\mathrm{Q}$ is the warmth source work and has been adjusted here to deal with variety of resistivity of copper with temperature. The warmth source term Q can be of the frame:

$Q=Q_{0} \cdot\left[1+\alpha_{c} \cdot\left(T-T_{a m b}\right)\right]$

Where, $\alpha \mathrm{c}$ is the temperature coefficient of electrical protection of copper wire. With this portrayal, the capacity $\mathrm{Q}$ progresses toward becoming temperature needy, conveyed warm source. Limit capacities $\mathrm{f1}(\mathrm{y})$ and $\mathrm{f} 2(\mathrm{y})$, got from Newton's law of cooling are of the frame (7) and (8):

$$
\begin{array}{r}
f_{1}(y)=h_{1} \cdot\left(T_{b}+m_{1} y\right) \\
f_{2}(y)=h_{2}\left(T_{b}+m_{2} y\right)
\end{array}
$$

The term $\mathrm{Tb}$ is the temperature at the base of the circle or layer, as appropriate. Terms $\mathrm{m} 1$ and $\mathrm{m} 2$ are the temperature slope along the twisting stature (for layer) or along circle thickness for a plate. Also, capacities $\mathrm{f} 3$ and f4representing temperature profiles crosswise over base and best surfaces, having the frame same as appeared in (7) and (8), where temperature angle term has been taken as zero:

$f_{3}(x)=h_{3} T_{b}$
Where:

$$
\begin{gathered}
k_{r}=\frac{\log \frac{r_{n}}{r_{1}}}{\left(\frac{\log \frac{r_{2}}{r_{1}}}{k_{1}}+\frac{\log \frac{r_{3}}{r_{2}}}{k_{2}}+\cdots+\frac{\log \frac{r_{n}}{r_{n-1}}}{k_{n}}\right)} \\
k_{z}=\frac{k_{c u} k_{i n}\left(t_{c u}+t_{i n}\right)}{\left(t_{i n} k_{c u}+t_{c u} k_{i n}\right)}
\end{gathered}
$$

Term $\mathrm{K}$ speaks to resultant warm conductivity of protection and conductor framework. Warmth exchange coefficients $\mathrm{hl}$ to $\mathrm{h} 4$ (htc), are distinctive over all the four surfaces. To decide limit capacities f1 to $\mathrm{f} 4$, it is important to compute warm exchange coefficients over the four surfaces. Trouble has been experienced in computation warm exchange relies upon upwards of 13 factors (e.g., winding size, type, conduit measurements, oil speed, and kind of oil flow, warm transition dispersion, oil warm properties, and so forth.). In this work, amendments have been given for temperature reliance of the warm and physical properties of oil, for example, thickness, particular warmth, volumetric extension and warm conductivity. It was discovered that there is immaterial impact of particular warmth, coefficient of volumetric extension and conductivity in the present working scope of stacking.

Following are a portion of the warmth exchange relations and significant formulae in normal cooling $(\mathrm{ON})$ mode. These formulae have been utilized to ascertain the htc. Nearby Nusselt, number for laminar stream over vertical plates has been demonstrated as follows:

$$
N u=0.6 R a_{h f}^{0.2}
$$
coefficient. It is accounted for somewhere else that it 
$R a_{h f}=G r_{h f} P_{r}$

Where Rahf and Grhf are the nearby Rayleigh and Grashof number in light of warmth transition (qw) at trademark measurement $(\delta)$.

$\operatorname{Pr}$ is the Prandtl number of transformer oil. Articulation of Rayleigh number in light of consistent warmth transition is:

$$
R a_{h f}=\frac{g \beta C_{p} \rho^{2} q_{w} \delta^{4}}{k_{o i l}^{2} \mu}
$$

Mean Nusselt number for this situation can be figured as:

$N u_{m}=1.25[N u]_{\delta=1}$

Notwithstanding, revision to recipe (14) must be given for tube shaped ebb and flow. The amendment factor for this situation is of the accompanying structure $(30<\operatorname{Pr}<50)$ :

$$
f(\xi)=1+0.12 \xi
$$

Where:

$$
\xi=\frac{2 \sqrt{2}}{G r^{0.25}} \times \frac{\delta}{r}
$$

Here $\mathrm{Gr}$ is Grashof number in view of temperature contrast. Warmth exchange coefficient can be figured as:

$h=\frac{N u k_{\text {oil }}}{\delta}$

Where, h speaks to neighborhood coefficient. Mean coefficient (hm) can be figured from mean Nusselt number, as in (15). In the wake of knowing hm for a specific surface, temperature contrast between the winding surface and oil can be discovered, partitioning the hm by the warmth motion through the surface. Mean Nusselt number of best surface of annular barrel shaped twisting for laminar and turbulent administration, will ordinarily be of the frame:

$$
\begin{aligned}
& N u_{m}=0.54 R a^{0.25}=0.61 R a_{h f}^{0.2} \\
& N u_{m}=0.15 R a^{\frac{1}{3}}=0.24 R a_{h f}^{\frac{1}{4}} \\
& \delta=\frac{b-a}{2}
\end{aligned}
$$

Where an and $b$ are the internal and external span of annular circle or layer. Nusselt number of base surface of annular barrel shaped twisting for laminar and turbulent administration is in (23):

$$
N u_{m}=0.27 R a^{0.25}=0.35 R a_{h f}^{0.2}
$$

Utilizing (24) can discover the pivotal oil temperature angle in nearness of cooling by a steady warmth motion, because of in this way:

$$
\frac{\partial T}{\partial y}=4.25 \times 10^{-2} \frac{q_{w}}{k_{\text {oil }}}\left(\frac{1}{\pi D_{m}}\right)^{\frac{4}{9}} R a_{h f}^{-\frac{1}{9}}
$$

Where 1 is the winding tallness, Dm is the mean width of annular plate or layer of windings. Assurance of limit conditions in constrained convection (OF mode) too requires ascertaining htc. The articulation when the oil speed is bring down qualities, the mean Nusselt number in light of temperature distinction is of the type of (25), comparing mean Nusselt number in view of consistent warmth transition is of the type of (26):

$$
\begin{aligned}
& N u_{m}=1.75\left[G_{z}+0.012\left(G_{z} G r^{\frac{1}{3}}\right)^{\frac{4}{3}}\right]^{\frac{1}{3}} \times\left(\frac{\mu_{b}}{\mu_{w}}\right)^{0.125} \\
& \text { Scientific } \\
& N u_{m}=1.63\left[1.8 G_{z}^{0.9}+0.02\left(G_{z} G r^{\frac{1}{3}} h f\right)^{1.16}\right]^{\frac{1}{3}} \times\left(\frac{\mu_{b}}{\mu_{w}}\right)^{0.125} \\
& C h \text { and } \\
& \text { onenen } G_{z}=\operatorname{RePr} \frac{D}{l}
\end{aligned}
$$

Where GzcalledGraetz number. The terms $b$ and $w$ are thickness of oil figured at oil mass mean temperature of oil and at winding divider temperature, individually. Re is the Reynolds number and $\operatorname{Pr}$ is the prandtl number. The relative significance of characteristic and constrained cooling is demonstrated by the factor $\mathrm{fr}=\mathrm{Gr} / \mathrm{Re} 2$. On the off chance, that fr 1 then both of the cooling modes must be considered. At a lower estimation of this factor, common cooling can be disregarded. The oil consistency is an imperative property, which relies upon temperature. The equation to take the oil temperature variety into thought is given in (28) underneath from:

$\mu=\alpha \cdot \exp \left(\frac{\gamma}{T_{m}}\right)$

Where:

$$
\begin{aligned}
& \alpha=0.0000013573\left(\mathrm{~kg} \cdot \mathrm{m}^{-1} \cdot \mathrm{s}^{-1}\right) \\
& \gamma=2797.3(\mathrm{~K})
\end{aligned}
$$


The thickness was figured at the mean oil and divider temperature. At first, the winding surface temperature isn't known, so a beginning estimate for winding surface temperature must be made, in the wake of computing the estimation of $\mathrm{h}$, the temperature contrast (Tw-Drudge) is to concur with the expected esteem. To figure winding divider temperature at various surfaces, just base oil temperature is essential. In this paper, the base oil ascend over surrounding temperature has been ascertained as:

$\theta_{u}=\theta_{f l} \cdot\left(\frac{I_{r}^{2} R+1}{R+1}\right)^{n}$

Where $u$ is the base oil temperature, ascend over encompassing Temperature $\mathrm{fl}$ is the full load base oil temperature ascend over surrounding temperature acquired from a disconnected test; $\mathrm{R}$ is the proportion of load misfortune at appraised load to no-heap misfortune. The variable Iris the proportion of the predefined load to evaluated stack:

$I_{r}=\frac{I}{I_{\text {rated }}}$

The type $\mathrm{n}$ relies on the cooling state. The stacking guide prescribes the utilization of $n=0.8$ for normal convection and $\mathrm{n}=0.9-1.0$ for constrained cooling.

Suppositions have been made in the estimation of htc in the channels gave in the plate write windings under oil-constrained (OF) methods of warmth exchange (DOF and NDOF). The cooling in OF mode is because of blended mode (common and constrained) convection. While the oil-stream speed in both vertical and level channels has been accepted equivalent, the mode is DOF. In the event that the stream speed in level conduits is expected irrelevant contrasted with speed in the vertical pipes, the mode is NDOF. If there should be an occurrence of DOF mode, the htc was accepted an element of both warmth transition through the surface and the oil stream speed. The system of warmth move in this method of cooling is same in both pivotal and spiral bearing of the plate. If there should arise an occurrence of NDOF mode, the htc in the vertical pipe has been assessed by an indistinguishable recipe from for DOF [5]. Nevertheless, the convection of warmth in the level pipes has been expected aregular compose.

\section{SIMULATION AND RESULTS}

The FEM of the transformer is performed to confirm the adequacy of the hypothetical conditions utilized as a part of the plan procedure and approve the outline parameters. The FEM for the situation examine includes these stages: attractive and warm field dispersion inside the transformer.

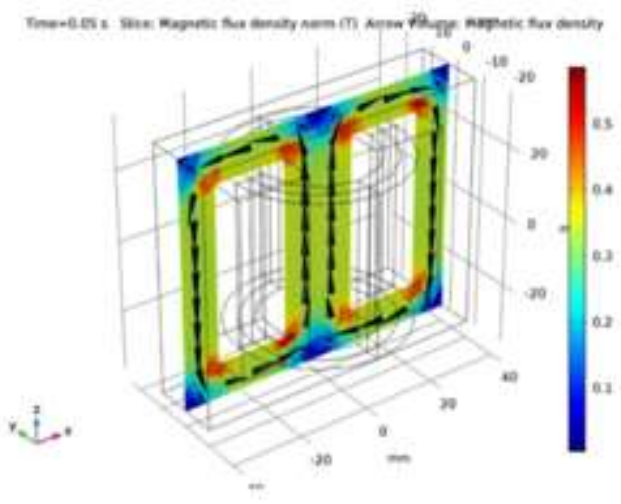

\section{A. Design parameters of the transformer}

To research the proposed approach, a transformer was composed. Center compose windings are utilized for the theoretical plan. The essential and auxiliary windings have $3 \mathrm{E} 5$ layers and each layer 300 turns. The principle parameters of the transformer are appeared in table 1 .

\section{TABLE I PARAMETERS OF TYPICAL} TRANSFORMER.

\begin{tabular}{|l|l|l|l|}
\hline Name & Expression & Value & Description \\
\hline Rp & $100[\mathrm{ohm}]$ & 100 & $\begin{array}{l}\text { Primary side } \\
\text { resistance }\end{array}$ \\
\hline Rs & $100[\mathrm{ohm}]$ & 100 & $\begin{array}{l}\text { Secondary side } \\
\text { resistance }\end{array}$ \\
\hline $\mathrm{Np}$ & $3 \mathrm{e} 5$ & $3 \mathrm{E} 5$ & $\begin{array}{l}\text { Number of turns } \\
\text { in primary } \\
\text { winding }\end{array}$ \\
\hline $\mathrm{Ns}$ & 300 & 300 & $\begin{array}{l}\text { Number of turns } \\
\text { in secondary } \\
\text { winding }\end{array}$ \\
\hline nu & $50[\mathrm{~Hz}]$ & $50 \mathrm{~Hz}$ & $\begin{array}{l}\text { Frequency of } \\
\text { Supply voltage }\end{array}$ \\
\hline Vac & $25[\mathrm{kV}]$ & $25000 \mathrm{~V}$ & Supply Voltage \\
\hline
\end{tabular}

\section{B. Simulations of magnetic field}

In this segment, the reenactment comes about acquired utilizing COMSOL Multiphysics programming [6] [7] is talked about. The shading plotting of the attractive field conveyance for the situation think about got because of the reenactment 
procedure is clarified. The shading plots of the attractive field conveyance acquired as a result of $2 \mathrm{D}$ reproductions is appeared in figure 1 . Alluding to the numerical motion thickness esteems appeared in the figure 1, the surface plot of the attractive flux thickness standard conveyance and the bolt plots of the streams in the windings at $\mathrm{t}=50 \mathrm{~ms}$.

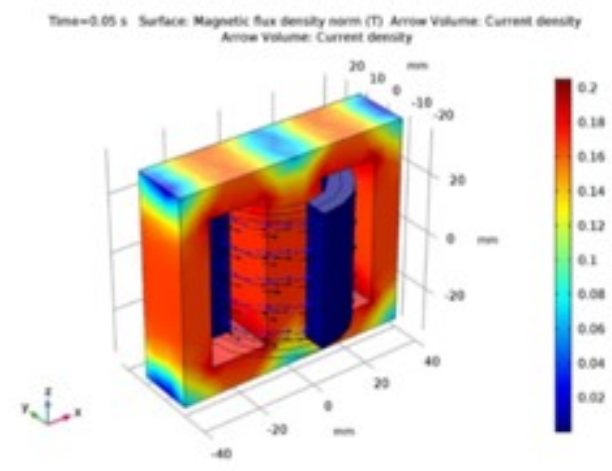

Fig-1: Magnetic flux density norm and the currents in the winding at $\mathrm{t}=50 \mathrm{~ms}$

\section{B.1. Simulation of magnetic flux density norm in the core at $\mathrm{t} z 50 \mathrm{~ms}$.}

The shading plots of the warm field appropriation acquired as a result of $2 \mathrm{D}$ reproductions is appeared in

Fig-2. Magnetic flux density inside the transformer at $\mathrm{t}=50 \mathrm{~ms}$

Figure 2. Attractive flux thickness standard in the center at $\mathrm{t} \mathrm{z} 50 \mathrm{~ms}$. it can be seen that, in figure 2 , the extreme estimation of the temperature is $136^{\circ} \mathrm{C}$.

\section{CONCLUSION}

In this paper, an endeavor has been made to propose a technique to enhance the exactness of forecast of the temperature of the most blazing spot in control transformer by unraveling the warmth exchange fractional differential condition (PDE) numerically. The simply numerical approach for assessing problem area and its area followed in this paper appears to compare sensibly well with the consequences of estimations and real tests and on location estimations. The creators wish to bring up that the IEEE stacking guide and other comparative records offer relations for the estimation of the HST in view of p.u. stack. The plans have a tendency to overlook the potential outcomes of two transformers that are appraising indistinguishable yet have an alternate winding structure and changing warmth misfortune/unit volume. The strategy proposed by the creators gives due portrayal for this exclusion and, subsequently, is accepted to give more precise evaluations.

\begin{tabular}{|l|l|l|l|}
\hline Type & $\begin{array}{l}\text { Stray } \\
\text { loss }\end{array}$ & $\begin{array}{l}\text { Hot } \\
\text { spot } \\
\text { factor }\end{array}$ & $\begin{array}{l}\text { Hotspot/winding } \\
\text { Temperature }\end{array}$ \\
\hline $\begin{array}{l}\text { Without } \\
\text { shielding }\end{array}$ & $11 \mathrm{Kw}$ & 1 & $69.94^{\circ} \mathrm{C}$ \\
\hline $\begin{array}{l}\text { With } \\
\text { shielding }\end{array}$ & $7.5 \mathrm{Kw}$ & 1.3 & $48.15^{\circ} \mathrm{C}$ \\
\hline
\end{tabular}

A warm model that can screen the warm profile of the transformer has been effectively displayed. A FEM display was adjusted and used to foresee the transformer precise stray misfortunes.

The learning of motion thickness was utilized with conductor measurements to foresee the whirlpool misfortunes for a particular outline. The model can be utilized to ascertain the vortex misfortune in singular turns/circles, to find the winding problem area, and to foresee the problem area factor H. Such data is vital for winding problem area assurance. The transition thickness plot of the casing shows that the curl side has extensive motion thickness than that of the tank side, and that the case with a shunt has a littler motion thickness dissemination, when contrasted with the case without. The table demonstrates the aggregate stray misfortunes in transformer without, and with, divider shunts. It can be seen that the decrease of stray misfortunes altogether lessens the misfortunes and problem area temperature and enhance the productivity and life expectancy of the transformer. The introduced demonstrate permits transient FE examination of transformers, which are coupled to outer hardware.

The transformer is displayed in the $\mathrm{mf}$ method of COMSOL Multiphysics 5.3a [8] [9], which settles for the attractive field.

The nonlinear polarization normal for the center material is represented in the shape $\mathrm{H}=\mathrm{f}(|\mathrm{B}|)$ eB in light of a worldwide objective guesswork. Transformer windings comprising of numerous turns are displayed as mass districts. In the essential conductor, swirl streams are considered by methods for a worldwide condition. Because of their better structure, skin impact can be dismissed in the auxiliary windings. The electromagnetic model likewise processes the time-found the middle value of 

energy misfortune thickness dissemination, which fills in as a contribution for the warm model.

\section{REFERENCES}

1. https://en.wikipedia.org/wiki/Transformer

2. http://www.electricenergyonline.com/show_article .php?article $=311$

3. http://www.ijtra.com/see/estimation of-problem area temperature-and-maturing of-a-transformer.pdf

4. Www.ijmer.com/papers/vol\%201\%20issue\%202/ AA012425429.pdf

5. web.mit.edu/kjb/www/Books/FEP_2nd_Edition_4 th_Printing.pdf

6. https://www.comsol.com/comsol-multiphysics

7. https://www.comsol.com/

8. www.comsol.co.in/display/e-center transformer14123

9. https://www.comsol.ru/gathering/string/.../models. acdc.ecore_transformer-20997.pdf 